\title{
Sinusoidal Steady-state Response Research Based on the Time Domain, S Domain and MATLAB Software
}

\author{
Liu Yuying \\ College of Communication and Electronics \\ Jiangxi Science \&Technology Normal University \\ Nanchang, China \\ e-mail: 502625690@qq.com
}

\begin{abstract}
Circuital sinusoidal steady-state analysis is the important content in such curriculums as Circuital Analysis and Signals and Systems.It is the essential knowledge for college students majoring in electric, signals and procession of signals, communication, etc.Studying the usage of time-domain method or complex frequency domain method, this paper uses such numerical computing method as solving differential equations or algebra equations to explore an approach that can analyse the sinusoidal steady response with the use of numerical computing function of MATLAB software and modeling simulation function.
\end{abstract}

Keywords-time domain;s domain;MATLAB;sinusoidal steady response

\section{INTRODUCTION}

The solution methods of Circuital sinusoidal steady-state response introduced in two ways in the course of circuit analysis, one is to establish the mathematical model of input - output - differential equation, With the help of the phasor method, we transform the differential equation into the algebraic equation, then solution the steady- state response phase, thereby solving the steady-state response.The other is phasor model method, the circuit model is transformed into a phasor model, we can get the steady state response by the phasor model. In signal and system course introduces three methods, There are the time domain method v the frequency domain method and the complex frequency domain method. The phasor method and the frequency domain method, the phasor model method and the complex frequency domain`s $\mathrm{S}$ domain model method have similar principle[1] [2].

As a kind of science and engineering calculation software, MATLAB software has powerful numerical calculation function, visual graphics, professional symbolic computation and visualization modeling simulation function, using MATLAB to solve circuit steady-state response, we can be freed from the cumbersome manual operation and derivation of the mathematical, because of MATLAB`s vivid visual graphics and interactive visualization modeling and simulation, the difficulty of solving.is reduced very well. This paper use figure 1 sinusoidal circuit as example, the switch is from 1 to 2 at $t=0$, with $u_{s}(t)$ is excitation, $i(t)$ is response, from establishing the circuit`s input - output math model, to solutioning the steady-state response, it discusses its time domain, $\mathrm{S}$ domain, MATLAB programming and simulation method[3] [5].

\author{
Yin Zhijian \\ College of Communication and Electronics \\ Jiangxi Science \&Technology Normal University \\ Nanchang, China \\ e-mail:86412656@qq.com
}

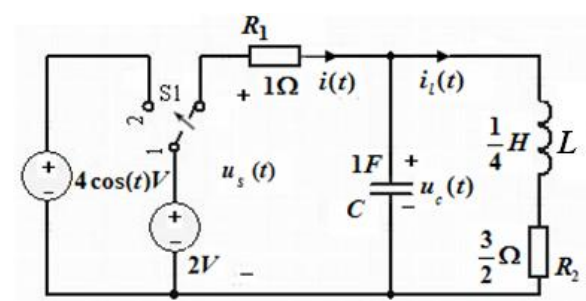

Figure 1. Curve: Sinusoidal Circuit

\section{ESTABLISHING CIRCUIT`S INPUT-OUTPUT RELATION MATHEMATICAL MODEL}

\section{A. Establishing differential equation on time domain method}

According to the circuit we can list the following equations:

$$
\left\{\begin{array}{l}
u_{s}(t)=2 \varepsilon(-t)+4 \cos (2 t) \varepsilon(t) \\
i(t)=C \frac{d u_{c}(t)}{d t}+i_{l}(t) \\
R_{1} i(t)+u_{c}(t)=u_{s}(t) \\
u_{c}(t)=L \frac{d i_{l}(t)}{d t}+R_{2} i_{l}(t)
\end{array}\right.
$$

After using elimination method eliminate $u c(t)$ and il(t), we can get:

$$
\begin{aligned}
& C L R_{1} \frac{d^{2} i(t)}{d t^{2}}+\left(\mathrm{L}+\mathrm{R}_{1} R_{2} C\right) \frac{d i(t)}{d t}+\left(R_{1}+R_{2}\right) i(t) \\
& =C L \frac{d^{2} u_{s}(t)}{d t^{2}}+C R_{2} \frac{d u_{s}(t)}{d t}+u_{s}(t)
\end{aligned}
$$

We substitute the circuit parameter to the above formula:

$$
\begin{aligned}
& \frac{d^{2} i(t)}{d t^{2}}+7 \frac{d i(t)}{d t}+10 i(t)=\frac{d^{2} u_{s}(t)}{d t^{2}}+6 \frac{d u_{s}(t)}{d t} \\
& +4 u_{s}(t)
\end{aligned}
$$

The equation is the circuit input-output description method mathematical model- differential equation。 


\section{B. Establishing differential equation on transform domain ( $s$ domain) method}

The first,we must establish circuit's s domain model, for the circuit's steady state response is forced response of zero state response of complete response, so we only need to establish circuit's s domain model in zero state, shown in figure 2 .

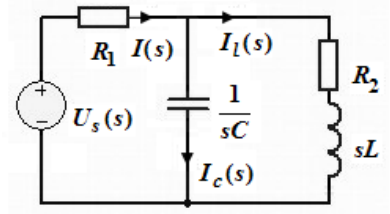

Figure 2. Circuit's s domain model in zero state

$$
\left\{\begin{array}{c}
I(s)=I_{c}(s)+I_{l}(s) \\
U_{s}(s)=I(s)+I_{c}(s) \frac{1}{s C} \\
U_{s}(s)=I_{s}(s) R_{1}+I_{l}(s)\left(s L+R_{2}\right)
\end{array}\right.
$$

Eliminate $\boldsymbol{I}_{c}(\boldsymbol{s})$ and $\boldsymbol{I}_{l}(\boldsymbol{s})$, we substitute the circuit parameter to the above formula:

$$
I(s)=\frac{s^{2}+6 s+4}{s^{2}+7 s+10} U_{s}(s)
$$

So system function:

$$
H(s)=\frac{s^{2}+6 s+4}{s^{2}+7 s+10}
$$

According to the system function and the system mathematical model of differential equations, We can derive mathematical model of circuit's input - output description method-differential equation:

$$
\begin{aligned}
& \frac{d^{2} i(t)}{d t^{2}}+7 \frac{d i(t)}{d t}+10 i(t)=\frac{d^{2} u_{s}(t)}{d t^{2}}+6 \frac{d u_{s}(t)}{d t} \\
& +4 u_{s}(t)
\end{aligned}
$$

\section{SOLVING THE CIRCUIT`S STEADY-STATE RESPONSE}

\section{A. The time domain method}

After establishing the mathematical model, the problem that solving the circuit's steady-state response is turned into the problem that solving the special solution of the differential equation in the zero state conditions and $t \geqslant 0$.

Substitute us(t) to formula,we can get:

$$
\begin{aligned}
& \frac{d^{2} i(t)}{d t^{2}}+7 \frac{d i(t)}{d t}+10 i(t)=2 \delta^{\prime}(t)+12 \delta(t) \\
& -48 \sin (2 t) \varepsilon(t)
\end{aligned}
$$

we can set special solution for:

$$
i_{p}(t)=k_{1} \sin (2 t)+k_{2} \cos (2 t)
$$

substitute to the above formula:

$$
\begin{aligned}
& -4 k_{1} \sin (2 t)-4 k_{2} \cos (2 t)+14 k_{1} \cos (2 t)-14 k_{2} \\
& \sin (2 t)+10 k_{1} \sin (2 t)+10 k_{2} \cos (2 t)=-48 \sin (2 t)
\end{aligned}
$$

we can get:

$$
\left\{\begin{array}{c}
6 k_{1}-14 k_{2}=-48 \\
6 k_{2}+14 k_{1}=0
\end{array}\right.
$$

solution for:

$$
\left\{\begin{array}{l}
k_{1}=-1.24 \\
k_{2}=2.90
\end{array}\right.
$$

so we can solve the special solution:

$$
\begin{aligned}
i_{p}(t) & =-1.24 \sin (2 t)+2.90 \cos (2 t) \\
& =3.15 \cos \left(2 t+23.2^{\circ}\right)
\end{aligned}
$$

The circuit`s steady-state response:

$$
i_{s s}(t)=3.15 \cos \left(2 t+23.2^{\circ}\right)
$$

\section{B. The transform domain ( $s$ domain) method}

Transform domain method is based on the system function, to find out the zero state response, then to separate the steady-state response from it; or Transform domain method is based on the circuit's differential equations, by The Laplace transform of differential equation in zero state we can solve Laplasse transform of zero state response, then separated the steady state response from among[4].

$$
\begin{aligned}
I_{z s}(s) & =H(s) U_{s}(s) \\
& =\frac{s^{2}+6 s+4}{s^{2}+7 s+10} \frac{4 s}{s^{2}+4} \\
& =\frac{1.10 s+6.21}{s^{2}+7 s+10}+\frac{2.90 s-2.48}{s^{2}+4}
\end{aligned}
$$

So the Laplasse transform of steady state response is:

$$
I_{p}(s)=\frac{2.90 s-2.48}{s^{2}+4}
$$

The circuit`s steady-state response:

$$
i_{\mathrm{ss}}(t)=3.15 \cos \left(2 t+23.2^{\circ}\right)
$$

\section{C. $\quad$ The method of steady state response based on $M A T L A B$}

The algorithm of the steady-state response based on MATLAB is also very rich, this paper will introduce the 
programming method and simulink graphical method.Before the two solutions are applied,we must use ss2tf function of MATLAB signal processing box to change the state space model that obtained by equations(1) into transfer function model, so we determine the system function, or differential equations.

Plug the data into equations(1) and transform it into:

$$
\left\{\begin{array}{c}
u_{c}^{\prime}(t)=-u_{c}(t)-i_{l}(t)+u_{s}(t) \\
i_{l}^{\prime}(t)=4 u_{c}(t)-6 i_{l}(t)+0 \cdot u_{s}(t) \\
i(t)=-u_{c}(t)+0 \cdot i_{l}(t)+u_{s}(t)
\end{array}\right.
$$

set the state variable : $\mathrm{X}=\left[\begin{array}{c}\boldsymbol{u}_{c}(t) \\ \boldsymbol{i}_{l}(t)\end{array}\right]$,

the input vari able : $U=\left[\boldsymbol{u}_{s}(t)\right]$

the output var iable : $Y=[i(t)]$

$A=\left[\begin{array}{cc}-1 & -1 \\ 4 & -6\end{array}\right], B=\left[\begin{array}{l}1 \\ 0\end{array}\right]$,

$C=\left[\begin{array}{ll}-1 & 0\end{array}\right] D=[1]$

so equations (17) can be writed into matrix form :

$\left\{\begin{array}{c}X^{\prime}=A X+B U \\ Y=C X+D U\end{array}\right.$

Equations(18) is the state space model, Call file lyy1, the state space model is converted into a transfer function model.

\%lyy1 the state space model is converted into a transfer function model

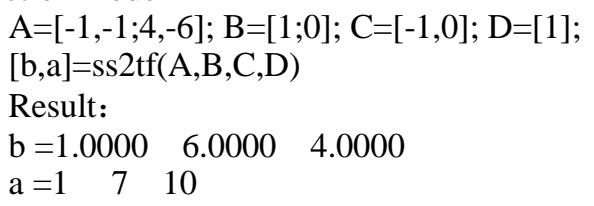

So we can conclude Circuit's system function and mathematical model :

$$
H(s)=\frac{s^{2}+6 s+4}{s^{2}+7 s+10}
$$

$$
\frac{d^{2} i(t)}{d t^{2}}+7 \frac{d i(t)}{d t}+10 i(t)=\frac{d^{2} u_{s}(t)}{d t^{2}}+6 \frac{d u_{s}(t)}{d t}+4 u_{s}(t)
$$

\section{1)The programming method based on MATLAB}

The programming method based on MATLAB is various, this paper introduces two methods, one is using the circuit 's zero state response waveform, observe the amplitude of steady-state portion and the time interval of Adjacent the steady-state response and Excitation Peak value, determine the steady state response. The other is According to the definition of the system frequency response :the system's input is a sinusoidal signal, the steady-state response is the same frequency sinusoidal signal, the amplitude is the
Product of the input signal 's amplitude and the frequency response's amplitude in the input signal's frequency, the phase is the and of the input signal 's phase and the system frequency response's phase in the input signal's frequency.By programming to solve the frequency response,we can get the amplitude and phase in the input signal`s frequency, to determine the steady state response[6].

\%lyy1 solve the circuit 's zero state response to determine the steady state response

$\mathrm{b}=\left[\begin{array}{lll}1 & 6 & 4\end{array}\right] ; \mathrm{a}=\left[\begin{array}{lll}1 & 7 & 10\end{array}\right]$;

sys $=\mathrm{tf}(\mathrm{b}, \mathrm{a}) ; \mathrm{p}=0.001 ; \mathrm{t}=0: \mathrm{p}: 10$;

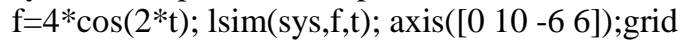

Result(figure 3):

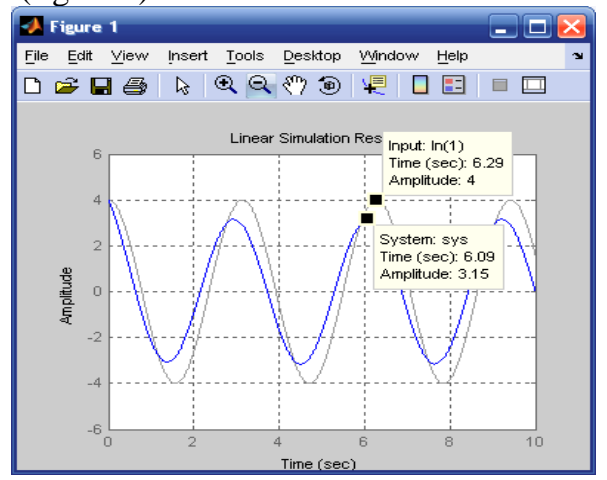

Figure 3. Circuit's response in zero state

Figure 3 is the input and output waveform, From the output waveform, we know the amplitude is 3.15 , The output phase dvance input signal 2 (6.29-6.09) rad / s, This is 0.40 $\mathrm{rad} / \mathrm{s}$,So the circuit`s steady-state respnse:

$$
\begin{aligned}
i_{s s}(t) & =3.15 \cos (2 t+0.40) \\
& =3.15 \cos \left(2 t+22.9^{\circ}\right)
\end{aligned}
$$

\%lyy2 applicate freqs function to solve frequency response and mapping to determine the steady state response

$\mathrm{b}=\left[\begin{array}{lll}1 & 6 & 4\end{array}\right] ; \mathrm{a}=\left[\begin{array}{lll}1 & 7 & 10\end{array}\right]$;

$\mathrm{w}=0: 0.1: 4 ; \mathrm{H}=$ freqs $(\mathrm{b}, \mathrm{a}, \mathrm{w})$;

$\mathrm{HM}=\mathrm{abs}(\mathrm{H})$

$\mathrm{HP}=$ angle $(\mathrm{H})$

subplot(221); plot(w,HM);

xlabel('Frequency $(\mathrm{rad} / \mathrm{s})$ ');

ylabel('Magnitude');

axis([0 40.2 1]); grid on;

subplot(222); plot(w,HP*180/pi);

xlabel('Frequency(rad/s)');

ylabel('Phase(degrees)');

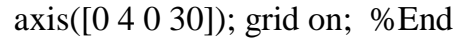

Result: ( the underlined part is frequency response's amplitude and phase in $2 \mathrm{rad} / \mathrm{s}$ )

$\mathrm{HM}=$

Columns 13 through 18

$\begin{array}{llllll}0.6372 & 0.6601 & 0.6819 & 0.7026 & 0.7220 & 0.7402\end{array}$

Columns 19 through 24

$\begin{array}{llllll}0.7572 & 0.7731 & \underline{0.7878} & 0.8016 & 0.8144 & 0.8262\end{array}$

$\mathrm{HP}=$ 
Columns 13 through 18

$\begin{array}{llllll}0.4532 & 0.4521 & 0.4488 & 0.4438 & 0.4375 & 0.4302\end{array}$

Columns 19 through 24

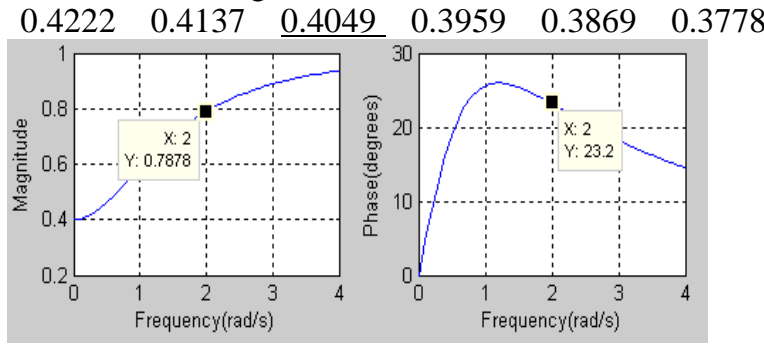

Figure 4. Circuit's amplitude spectrum and phase spectrum

The numerical solutions available from frequency response, the circuit's steady-state response:

$$
\begin{aligned}
i_{s s}(t) & =4 \times 0.7878 \cos (2 t+0.4049) \\
& =3.15 \cos \left(2 t+23.2^{\circ}\right)
\end{aligned}
$$

From figure 4 the frequency response's amplitude spectrum and phase spectrum, we can get the circuit's steadystate response:

$$
\begin{aligned}
i_{s s}(t) & =4 \times 0.7878 \cos \left(2 t+23.2^{\circ}\right) \\
& =3.15 \cos \left(2 t+23.2^{\circ}\right)
\end{aligned}
$$

\section{2)The simulation method based on MATLAB}

Figure 5 is a block diagram of system simulation, the above of figure 6 is the input signal waveform, the below of figure 6 is the output signal waveform. by amplifying the output waveform, we get the last amplitude of the output signal waveform is 3.09 , and we measured the moments that two waveforms cross the horizontal axis finally a rising process are about $18.83 \mathrm{~s}$ and $18.62 \mathrm{~s}$, the Output phase Advances the input phase about 0.42rad / s, Angle is about 24 degrees. So the circuit's steady-state response:

$$
i_{s s}(t)=3.09 \cos \left(2 t+24^{\circ}\right)
$$

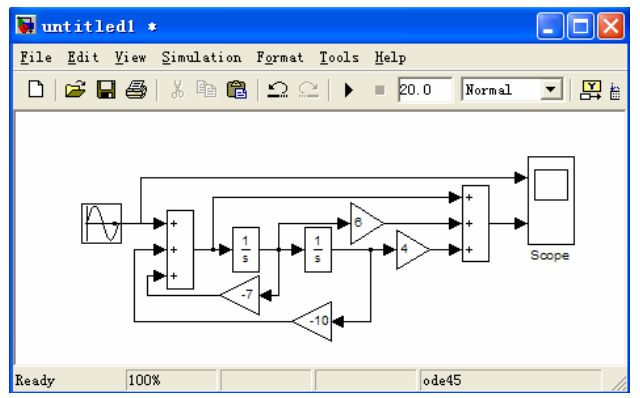

Figure 5. Circuit' s simulation block diagram

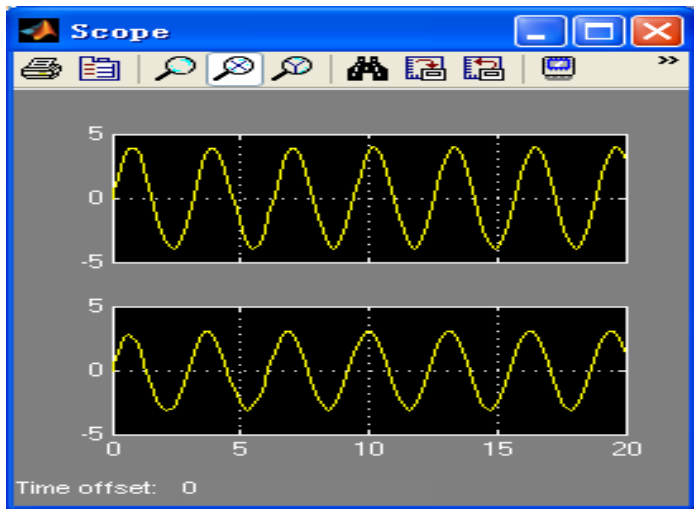

Figure 6. The input and output waveforms of simulation system

\section{CONCLUSIONS}

Sinusoidal steady-state analysis is important content in circuit analysis and signal and system course, it is the knowledge points that the electronic, signal processing and communication professionals`s students must master. What this paper summarizes are not all methods, but it should be expressed the basic concepts and methods of analysis of sinusoidal steady state analysis. I don't think that way is better, I think that in the engineering application software analysis method is better. But to undergraduate students, they must master the simple system's manual analysis processhe of the time domain、s domain analysis method, through this process, they can understand the principles truly. In addition to the above analysis method, we also can be directly to simulation circuit in the MATLAB Simulink environment. With new technology 、 new software's emerge in large numbers, we must continuous exploration to discover more and better methods.

\section{REFERENCES}

[1] Li Hansun. Basis of Circuit Analysis(3rd edition). Beijing: Higher Educational Press,1994,pp.35-42.

[2] Zheng Junli, Ying Qixian, Yang Weili. Signals and Systems.(3rd edition). Beijing: Higher Educational Press,2000,pp.224-230.

[3] Wu Dazheng, Yang Linyao, Zhang Yongrui, Wang Songlin, Guo Baolong. Signal and Linear System Analysis(4th edition). Beijing: Higher Educational Press,2005,pp.243-257.

[4] Michaels,Roberts,Sigmals and Systems. Hu Jianling,eds. Beijing: China Machine Press,2006,pp.312-317.

[5] Guan Zhizhong, Xia Gongke, Meng Qiao. Signals and Linear System(4th edition). Beijing: Higher Educational Press,2004,pp.198205.

[6] Meret Oppenheim A.V eds. Signals and Systems(2nd edition). Liu Haitang translate. Xi'an: Xi'an Jiaotong University Press, 2000,pp. 254-262. 